\title{
TREATMENT OF GINGIVAL MELANIN PIGMENTATION USING CRYOSURGERY- Series of Case Reports
}

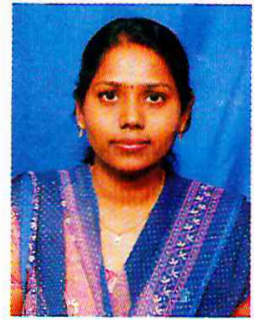

Dr. Radhika B. P.G. Student

\author{
Dept. of Periodontics
}

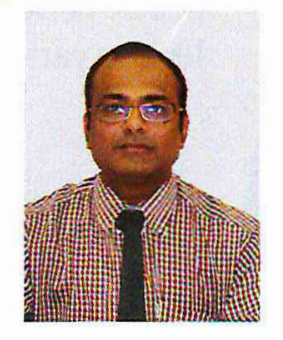

Dr. Narayan N. Walavalkar Professor

\section{ABSTRACT}

A smile expresses a feeling of joy, success, sensuality, affection and courtesy and reveals self confidence and kindness. The harmony of the smile is determined not only by the shape the position and the color of the teeth but also by the gingival tissues, gingival health and appearance are essential components of an alternative smile.

Although melanin pigmentation of the gingiva is completely benign and does not present a medical problem complaints of black gums are common particularly in patients having a very high smile line (gummy smile). To answer the cosmetic demand for a pleasing smile this paper describes cryosurgery technique \& scraping technique for elimination of dark pigmentation. The result of our present study showed short term benefit with cryosurgery with recurrence of pigmentation at 6 months. In conclusion cryosurgery gives a beneficial answer to cosmetic demand of pleasing smile.

\section{INTRODUCTION}

Melanin is normally found in the skin of people all over the world. However the amount varies considerably among people of different races. Oral melanin pigmentation is well documented in the literature and is considered to have multifactorial etiologies including genetic factors, tobacco use, systemic disorders and administration of certain drugs especially antimalarial agents and tricyclic antidepressant. ${ }^{4}$

Because dark gingival pigmentation is undesirable to some persons, it may well cause psychological problems for these patients, especially those to whom appearance is of vital importance..

Demand for de pigmentation is usually made for esthetic reasons, particularly in patients having a very high or high smile line. However there is not much information in the literature about de pigmentation of gingiva. Elimination of these melanotic areas through surgery, lasers, cryosurgery through the use of a gas expansion system, bur abrasion, scrapping and electrocautery have been reported each technique has its own advantages and inadequacies. ${ }^{1}$ 


\section{Material and Methods :}

The patients for this proposed study were selected from out patient Department of Periodontics, College ofDental Sciences, Davangere.

Ten patients, both males \& females aged between 15-30 yrs were selected. As esthetic is the prime factor in our study anterior segment is the site of choice in which gingiva of three teeth of one side was treated with cryosurgery and remaining gingiva of three teeth on another side was treated with scraping.

\section{Scraping}

After infiltration with anesthesia, blade no 11 with BP handle was used to scrape the epithelium with underlying pigmented layer. The raw surface is irrigated with saline solution. The surface is cleaned \& bleeding stopped. The exposed depigmented area is covered with a periodontal dressing for 1 week.

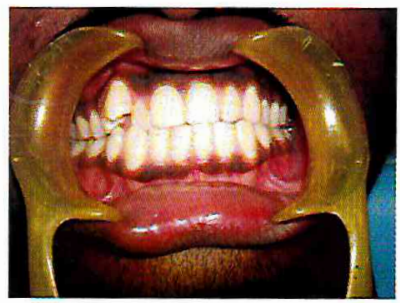

Pre-operative

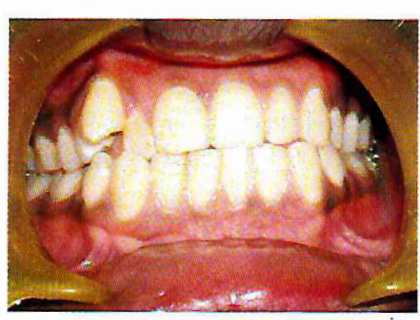

Post-op 6 months

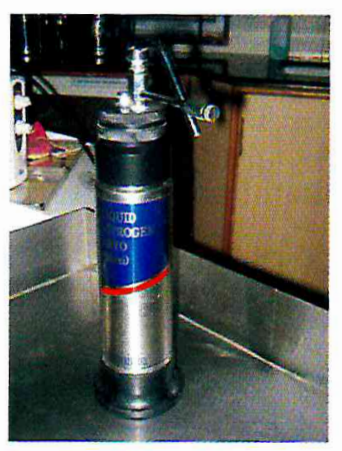

MINI CRYO GUN

\section{Cryosurgery}

After topical anesthesia direct application of liquid nitrogen $\left(-190^{\circ} \mathrm{c}\right)$ using MINI CRYO GUN liquid nitrogen cryo system model-LNC-196 is directly sprayed on to the gingiva till the ice ball is formed. No dressing was given and the patient was evaluated at 1 month, 3months, 6 months \& 9months.

\section{RESULTS}

No postoperative pain, hemorrhage, infection or scarring occurred on both sites. Patient acceptance of cryosurgery was good $\&$ results were excellent as perceived by the patient. There was repigmentation on cryosurgery site after 6 months.

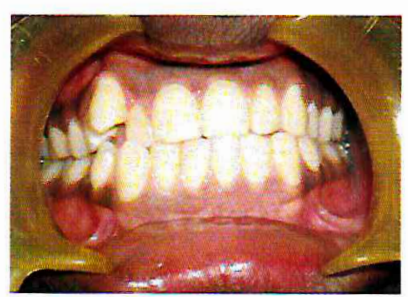

Post-op 9 months

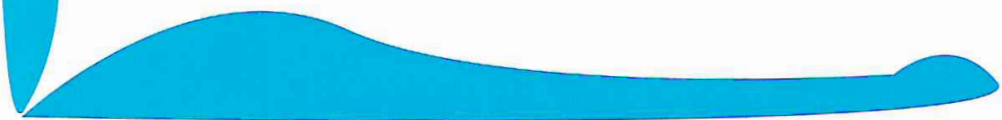




\section{DISCUSSION}

Cryosurgery, an effective method of tissue destruction by freezing, has become a firmly established surgical technique in medical and dental practice. Superficial gingival cryosurgery as well as full thickness gingival freezing in humans and animals have demonstrated healing by "complete regeneration" and "sterile inflammatory reaction. The main advantages were that no anesthesia was required, absolutely no hemorrhage hence chances of postoperative infection were minimal or nil, uneventful healing and easy technique. ${ }^{3}$

A free gingival graft which can be used to eliminate the pigmented area requires an additional surgical site and color matching and the presence of demarcated line commonly observaed around the graft in the recipient site may itself pose an esthetic problem.

A one step laser treatment is usually sufficient to eliminate the melanotic area.

Repigmentation is described as spontaneous and has been attributed to the activity and migration of cells from surrounding areas.

\section{SUMMARY}

Growing esthetic concern demand necessitates removal of unsightly gingival pigmentation or discoloration areas to create a pleasant and confident smile, which altogether may alter the personality of an individual. This can be easily achieved by cryosurgery which produced desired short term beneficial results and patient satisfaction which is the ultimate goal of any therapy that is carried out.

\section{REFERENCES}

1) Deepak Prasad, S Sunil, $\mathbf{R}$ Mishra, Sheshadri. Treatmentt of gingival Pigmentation: A Case series. Indian Journal of Dental Research 2005; 16: 171- 176.

2) Tal H, Landsberg $\mathbf{J}$ and Kozlovsky $\mathbf{A}$. Cryosurgical depigmentation of the gingiva- a case report. J Clin Periodontol 1987; 14 : 614-617.

3) Haim Tal. A novel cryosurgical technique for gingival depigmentation:Journal of the American Academy of Dermatology.

4) Vandana.K.L.,karthik.M. Treatment of gingival pigmentation: Case Reports. Karnataka State Dental journal 2004;23:45-47 